\title{
Reducing travel-related SARS-CoV-2 transmission with layered mitigation measures: symptom monitoring, quarantine, and testing
}

Michael A. Johansson * Đi, Hannah Wolford, Prabasaj Paul, Pamela S. Diaz, Tai-Ho Chen, Clive M. Brown, Martin S. Cetron and Francisco Alvarado-Ramy

\begin{abstract}
Background: Balancing the control of SARS-CoV-2 transmission with the resumption of travel is a global priority. Current recommendations include mitigation measures before, during, and after travel. Pre- and post-travel strategies including symptom monitoring, antigen or nucleic acid amplification testing, and quarantine can be combined in multiple ways considering different trade-offs in feasibility, adherence, effectiveness, cost, and adverse consequences.

Methods: We used a mathematical model to analyze the expected effectiveness of symptom monitoring, testing, and quarantine under different estimates of the infectious period, test-positivity relative to time of infection, and test sensitivity to reduce the risk of transmission from infected travelers during and after travel.

Results: If infection occurs 0-7 days prior to travel, immediate isolation following symptom onset prior to or during travel reduces risk of transmission while traveling by 30-35\%. Pre-departure testing can further reduce risk, with testing closer to the time of travel being optimal even if test sensitivity is lower than an earlier test. For example, testing on the day of departure can reduce risk while traveling by 44-72\%. For transmission risk after travel with infection time up to 7 days prior to arrival at the destination, isolation based on symptom monitoring reduced introduction risk at the destination by $42-56 \%$. A 14-day quarantine after arrival, without symptom monitoring or testing, can reduce post-travel risk by $96-100 \%$ on its own. However, a shorter quarantine of 7 days combined with symptom monitoring and a test on day 5-6 after arrival is also effective (97--100\%) at reducing introduction risk and is less burdensome, which may improve adherence.

\footnotetext{
* Correspondence: mjohansson@cdc.gov

The findings and conclusions in this report are those of the authors and do not necessarily represent the views of the Centers for Disease Control and Prevention. COVID-19 Response, Centers for Disease Control and Prevention, Atlanta, USA
}

(c) The Author(s). 2021 Open Access This article is licensed under a Creative Commons Attribution 4.0 International License, which permits use, sharing, adaptation, distribution and reproduction in any medium or format, as long as you give appropriate credit to the original author(s) and the source, provide a link to the Creative Commons licence, and indicate if changes were made. The images or other third party material in this article are included in the article's Creative Commons licence, unless indicated otherwise in a credit line to the material. If material is not included in the article's Creative Commons licence and your intended use is not permitted by statutory regulation or exceeds the permitted use, you will need to obtain permission directly from the copyright holder. To view a copy of this licence, visit http://creativecommons.org/licenses/by/4.0/. The Creative Commons Public Domain Dedication waiver (http://creativecommons.org/publicdomain/zero/1.0/) applies to the data made available in this article, unless otherwise stated in a credit line to the data. 
(Continued from previous page)

Conclusions: Quarantine is an effective measure to reduce SARS-CoV-2 transmission risk from travelers and can be enhanced by the addition of symptom monitoring and testing. Optimal test timing depends on the effectiveness of quarantine: with low adherence or no quarantine, optimal test timing is close to the time of arrival; with effective quarantine, testing a few days later optimizes sensitivity to detect those infected immediately before or while traveling. These measures can complement recommendations such as social distancing, using masks, and hand hygiene, to further reduce risk during and after travel.

Keywords: SARS-CoV-2, COVID-19, Travel, Testing, Quarantine

\section{Background}

Coronavirus disease 2019 (COVID-19) was first recognized in late December 2019. By March 2020, the virus causing COVID-19, SARS-CoV-2, had reached 6 continents and almost 70 countries. In response to the global COVID-19 outbreak, governments implemented a variety of mitigation measures including unprecedented social distancing measures, travel health alerts, and travel restrictions at national and sub-national levels $[1,2]$. These measures, as well as concern about exposures related to travel, led to major and prolonged reductions in air travel worldwide [3-7]. Spatiotemporally asynchronous waves of COVID-19 have led to dynamic risk and mitigation measures globally with an accompanying interest in identifying risk management steps for travel that can reduce the risk of transmission and address concerns of travelers, travel industry regulators, and public health authorities [8-10].

Initial policies for managing translocation of the virus from one destination to another relied on closing borders or restricting entry of travelers from countries with higher incidence rates $[11,12]$. Although these approaches may have reduced the importation of some cases and preserved resources, they came with enormous economic and individual impacts $[13,14]$.

For travelers, personal mitigation actions include wearing masks, social distancing at least $6 \mathrm{ft}$ from others when possible, frequent hand washing or use of alcoholbased hand sanitizer, not touching their face, and avoiding anyone who is sick. Governments, airlines, airports, and other businesses serving travelers have implemented or recommended measures to reduce the risk of COVID-19 associated with air travel [15, 16]. These measures have included enhanced disinfection procedures, employee health assessments, passenger health attestations, screening for fever, illness response protocols, increased spacing between passengers on flights, and other steps to reduce risk of transmission in airports and on conveyances [10,17]. Symptom-based screening at airports has proven ineffective because those measures miss mild, afebrile, asymptomatic, and pre-symptomatic SARS-CoV-2 infections [18-21]. Asymptomatic persons may account for $20 \%$ to $40 \%$ of SARS-CoV-2 infections and can transmit the virus to others [22-27], and epidemiological data indicate that infectiousness begins prior to symptom onset for those who do develop symptoms [28-32].

In many destinations, arriving travelers, most of whom are asymptomatic with no specific known exposures, were asked to self-quarantine and reduce contacts as much as possible after arrival. The World Health Organization (WHO) defines quarantine as "the restriction of activities and/or separation from others of the suspect persons... who are not ill, in such a manner as to prevent the possible spread of infection" and indicates that quarantine may be considered for travelers based on risk assessment and local conditions. For known SARS-CoV-2 exposures, WHO recommends quarantine of 14 days from their last exposure based on the limit of the estimated incubation period for SARS-CoV-2 [33]. A 14-day quarantine alone, when implemented immediately post-exposure and strictly adhered to, approaches $100 \%$ reduction in post-exposure transmission risk [34, 35]. However, travelers may have little incentive to consistently adhere to these measures at their destinations unless there is the ability to reliably communicate with them, support their needs, and enforce these measures. Monitoring and enforcing adherence to quarantine measures requires tremendous effort and resources by public health entities that may only be feasible and appropriate in certain contexts [36, 37].

Inclusion of SARS-CoV-2 testing as a component of a multi-layered approach to risk-reduction is currently being implemented in various settings. Some businesses and educational institutions are incorporating SARS-CoV-2 screening strategies into their concepts of operations, sometimes including mandatory testing of employees and voluntary testing for customers [38-40]. While there is no current international standard for testing travelers, many countries and jurisdictions are requiring arriving travelers to be tested either prior to their departure or after arrival to identify infected persons who are asymptomatic so they can be isolated [41]. Current guidance or requirements vary from country to country, and from state to state within the USA, including the timing of the 
test prior to or after travel, the type of test used (viral antigen, viral RNA), and the use of negative test results to alleviate additional public health measures, such as quarantine, at the destination [42, 43].

Currently available SARS-CoV-2 tests for detecting active infections include nucleic acid amplification tests (NAAT), such as reverse transcription polymerase chain reaction (RT-PCR) tests, rapid isothermal NAATs, and antigen-based tests. Time to deliver results is hours to days for RT-PCR and minutes to hours for antigen tests, which can also be processed without a specialized laboratory. Several antigen tests for SARS-CoV-2 are currently authorized in the United States for suspected SARS-CoV-2 infection $[44,45]$. While rapid antigen tests have advantages over NAATs in terms of cost, simplicity, and turnaround time, they are less likely to detect a positive in individuals with low viral load, i.e., early or late in infection [46]. However, the limited available data on the efficacy of antigen testing in asymptomatic individuals suggests they may have high sensitivity for infectious individuals [46-49].

SARS-CoV-2 transmission risk related to travel can be viewed in two domains: transmission risk during travel (e.g., by infected travelers while at an airport or on aircraft) and after travel is completed (e.g., introduction or re-introduction of SARS-CoV-2 to the destination location). There is also overlap as transmission risk during travel can lead to new infections, which can increase post-travel risk. Data on strategies for reducing risk associated with travel are scant and there are many potential strategies (e.g., the optimal timing of pre-departure or post-arrival testing or the combination of testing and post-arrival quarantine) [39, 50-52]. Mathematical models have provided some insights to the potential impact of quarantine combined with testing $[51,53]$. Here, we build upon those models, considering uncertainty in infectious periods and different testing options to assess a suite of possible combined pre- and post-travel strategies to reduce transmission risk from infected travelers.

\section{Methods}

First, we characterized component processes related to transmission risk during infection: the relative infectiousness over the course of infection, the proportion of infections resulting in symptoms, the timing of symptom onset for those who have symptoms, and the probability of testing positive over the course of infection.

We used three distinct models to characterize relative infectiousness over time, $I(t)$, specifying each as a density function of daily infectiousness such that the total infectiousness is equal to one and the curves only differ in the temporal distribution of transmission risk (Fig. 1a). We used a Gamma density function to approximate a 10-day infectious period with peak infectiousness on day
5 based on observations from numerous studies [27, 5458]. We also replicated a within-host infection model by Goyal et al. [59] by simulating infections in 10,000 individuals and recording the probability of being infectious at time steps of 0.1 days. We then fitted a density function to the set of times when these individuals were infectious. This indicated that most people are infectious from days 3 to 7 after the time they were infected, with tapering afterwards. The final model characterizes simulated infectious periods from Clifford et al. [51], based on estimated latent periods (the delay between infection and becoming infectious) [54] and infectious periods [60]. We simulated 10,000 individual-level paired latent and infectious periods then fit an empirical density function to the infectious time points. The Gamma model represents a simple assumption that does not capture individual level variability; however, both the Goyal et al. and Clifford et al. infectiousness models capture the population-level impacts of individual-level variability such that estimates based on these models may more completely reflect the potential impact across many individuals.

We assumed that $70 \%$ of all infections result in symptomatic COVID-19 cases [25], $\sigma_{0}$, and provided several additional sensitivity estimates assuming that $50 \%$ of infections result in symptoms. For the incubation period, $\sigma(t)$, we used a meta-estimate with a median of 5 days and a Log-Normal distribution based on a meta-analysis by McAloon et al. [61].

For diagnostic testing, $\rho(t)$, we used two models: one directly estimating positivity by RT-PCR and one approximating an antigen detection assay (Fig. 1b). For the RT-PCR model, we used the model generated by Clifford et al. [51] based on data from Kucirka et al. [62]. To approximate an antigen detection assay, we assumed that the assay would have $80 \%$ sensitivity, $s$, for infectious individuals [46-49] and scaled the probability of testing, $\rho$, to match the time-course of each infectiousness curve with a peak at $80 \%$ :

$$
\rho(t)=s I(t) / \max (I) .
$$

To assess the impact of test sensitivity we also compared this to a $95 \%$ sensitivity version of the same model.

We then constructed a model capturing these components to assess the impacts of testing, symptom monitoring, and quarantine (Table 1). Infections resulting in travel-related risk could occur before or during a trip and we use one of the infectiousness density functions described above, $I(t)$, which defines relative infectiousness at time $t$ relative to travel based on infection at time $\tau$ relative to travel (prior to travel is negative): 
A

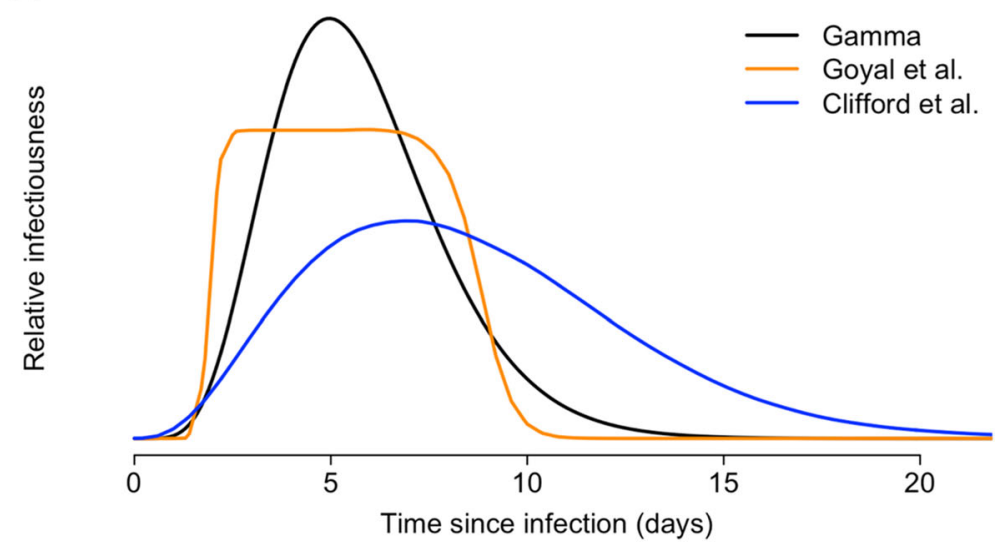

B

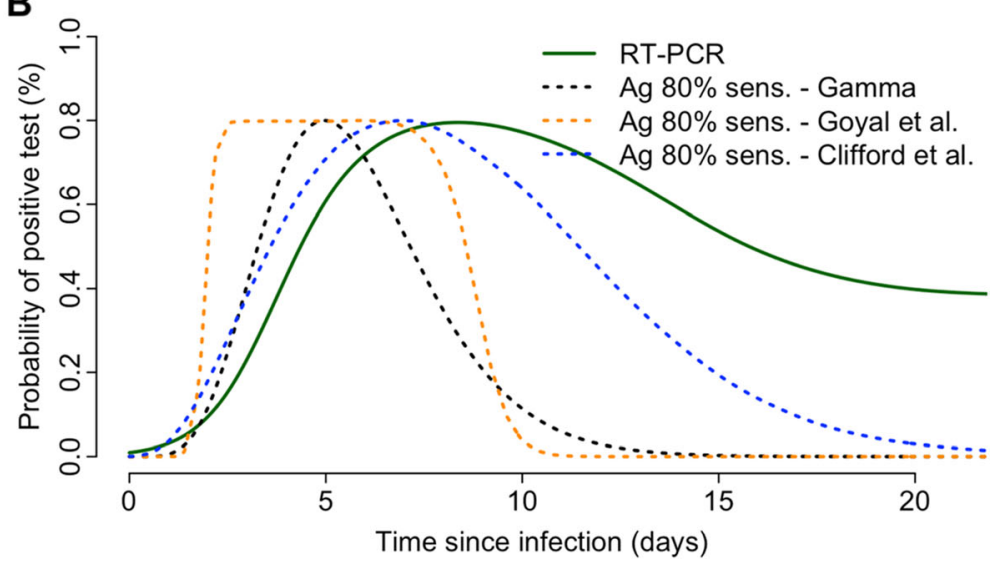

Fig. 1 Models of relative infectiousness and the probability of testing positive relative to time since SARS-CoV-2 infection. a Infectiousness density functions for a Gamma density function approximating a 10-day infectious period with a peak on day 5 [54-58], a host infection model adopted from Goyal et al. [59], and simulated infectious and latent periods adopted from Clifford et al. [51]. b Models of the probability of a positive test for SARS-CoV-2 relative to time since infection: a distribution estimating positivity by RT-PCR adopted from Clifford et al. [51] and antigen ("Ag") testing curves for each infectiousness curve (a) scaled such that test positivity tracks infectiousness with a maximum sensitivity of $80 \%$ at peak infectiousness

$$
I(t, \tau)=I(t-\tau) .
$$

Symptom monitoring was assessed as a method to detect and isolate infected individuals and therefore prevent transmission after symptom onset. As described above, we assumed that a proportion of infected individuals develop symptoms $\left(\sigma_{0}\right)$ and develop symptoms at rate $\sigma(t)$ as defined by the incubation period (described above). The onset of symptoms was assumed to lead to isolation until recovery, resulting in a residual in transmission risk over the transmission window:

$$
r_{S}(t, \tau)=1-\sigma_{0} \sigma(t-\tau) .
$$

Transmission at a time $t$ can also be mitigated through quarantine. We estimated the impact of quarantine as a reduction in risk of a magnitude equal to the adherence ${ }^{\alpha_{Q}}(1=100 \%)$ during a quarantine of duration $T_{Q}$ starting at the time of arrival $t_{0}$ with residual transmission risk:

$$
r_{Q}(t)=1{ }^{-}{ }_{Q} \text { if } t \in\left[t_{0}, t_{0}+T_{Q}\right], \text { and } 1 \text { otherwise. }
$$

Transmission can also be mitigated through test-based detection followed by isolation. For the purposes of the model, we assumed that test results were immediately available and a positive test immediately led to isolation until recovery. Test positivity for each test (described above) was characterized $\rho(t)$ and the corresponding residual in transmission associated with each test $k$ at test time $t_{k}$ is:

$$
r_{T}\left(t, \tau, t_{k}\right)=1-\rho\left(t_{k}-\tau\right) \text { if } t \in\left[t_{k}, \infty\right] \text {, and } 1 \text { otherwise. }
$$

For a set of tests, $K$, the residual risk is the product: 
Table 1 Model parameters

\begin{tabular}{lll}
\hline Variable & Definition & Values used \\
\hline$t$ & Time of transmission risk from traveler relative to time of travel & $\begin{array}{l}\text { Ranges: 0-1 days while traveling, 0-28 days } \\
\text { after travel }\end{array}$ \\
$\tau$ & Time of infection risk to traveler relative to time of travel & $\begin{array}{l}\text { or ranges: 1-7 days pre-departure, 1-7 days } \\
\text { pre-arrival }\end{array}$ \\
$I(t, \tau)$ & Infectiousness & Functions shown in Fig. 1a [27, 51, 59] \\
$\sigma_{0}$ & Proportion of infected individuals that develop symptoms & $70 \%, 50 \%$ \\
$\sigma(t, \tau)$ & Cumulative probability of developing symptoms among & Log-Normal cumulative distribution function [61] \\
$t_{0}$ & individuals who develop symptoms & \\
$\alpha_{Q}$ & Time of quarantine relative to the time of travel & 0 \\
$T_{Q}$ & Adherence to quarantine & $100 \%, 50 \%$ \\
$t_{k}$ & Duration of quarantine starting at $t_{0}$ & $7,10,14$ days \\
$\rho\left(t_{k}, \tau\right)$ & Time of test $k$ & 6 days pre-departure to 7 days post-arrival \\
$\epsilon(\tau)$ & Probability of a positive test & Functions shown in Fig. 1b \\
\hline
\end{tabular}

$$
r_{T}^{K}(t, \tau)=\prod_{k=1}^{K} r_{T}\left(t, \tau, t_{k}\right)
$$

Here, we assessed two transmission windows: days 01 for risk during travel to include potential risk in transit prior to and after airline travel and days 0-28 for risk after travel. The total transmission risk between times $t_{1}$ and $t_{2}$ for individuals infected at time $\tau$ is:

$$
I_{0}(\tau)=\int_{t_{1}}^{t_{2}} I(t, \tau) d t
$$

The transmission risk prevented by protocols including symptom monitoring, quarantine, and testing is:

$$
I_{S Q T}^{K}(\tau)=\int_{t_{1}}^{t_{2}} I(t, \tau)\left(1-r_{S}(t, \tau) r_{Q}(t) r_{T}^{K}(t, \tau)\right) d t
$$

For exposure windows in which a unique time of exposure is unknown, we assumed that infection may have occurred at any time in that window with equal probability. We therefore define the risk of exposure $\epsilon(\tau)$ as uniformly distributed over a window defined by the beginning and end of the exposure period, $\tau_{1}$ and $\tau_{2}$, respectively:

$$
\epsilon(\tau)=1 /\left(\tau_{2}-\tau_{1}\right) \text { if } \tau \in\left[\tau_{1}-\tau_{2}\right], \text { and } 0 \text { otherwise. }
$$

For example, with a 1-day trip, infection between 7 days pre-departure and the time of departure can be modeled relative to the time of arrival with $\tau_{1}=-8$ and $\tau_{2}=-1$.

Total infectiousness is then:

$$
I_{0}=\int_{\tau_{1}}^{\tau_{2}} \int_{t_{1}}^{t_{2}} I(t, \tau) d t d \tau
$$

The prevented transmission risk is:

$$
\begin{aligned}
I_{S Q T}^{K}= & \int_{\tau_{1}}^{\tau_{2}} \int_{t_{1}}^{t_{2}} \epsilon(\tau) I(t, \tau) \\
& \times\left(1-r_{S}(t, \tau) r_{Q}(t) r_{T}^{K}(t, \tau)\right) d t d \tau .
\end{aligned}
$$

Finally, we calculate the proportional reduction in transmission risk as: $I_{S Q T}^{K} / I_{0}$.

All analyses were conducted in $\mathrm{R}$ and the code is available at https:/github.com/cdcepi/COVID-19-traveler-model.

\section{Results}

Reducing transmission risk after a specific known exposure

Before looking at exposure over a range of times, we first assessed the impact of symptom monitoring, quarantine, and testing when the time of infection was known (for example, a brief high-risk contact). Isolating infected individuals at the time of symptom onset, without testing or quarantine, resulted in a reduction in transmission risk of $36-52 \%$ (minimum to maximum) accounting for differences in infectiousness over time between models relative to the onset of symptoms and an assumption that $30 \%$ of infected individuals never develop symptoms. If the proportion of individuals who never have symptoms was higher, the effect of symptom monitoring decreased. For example, if $50 \%$ of individuals never had symptoms, the reduction from symptom monitoring decreased to $26-37 \%$. Quarantine alone implemented immediately following exposure led to higher reductions in transmission risk, from 39 to $75 \%$ with 7 days to $90-$ $100 \%$ with 14 days. Isolating individuals based on a single positive test result alone produced a $0-67 \%$ reduction in transmission, depending on the day of the test relative to the infectious period and the time-specific test sensitivity (Fig. 2). Testing earlier in infection was less 


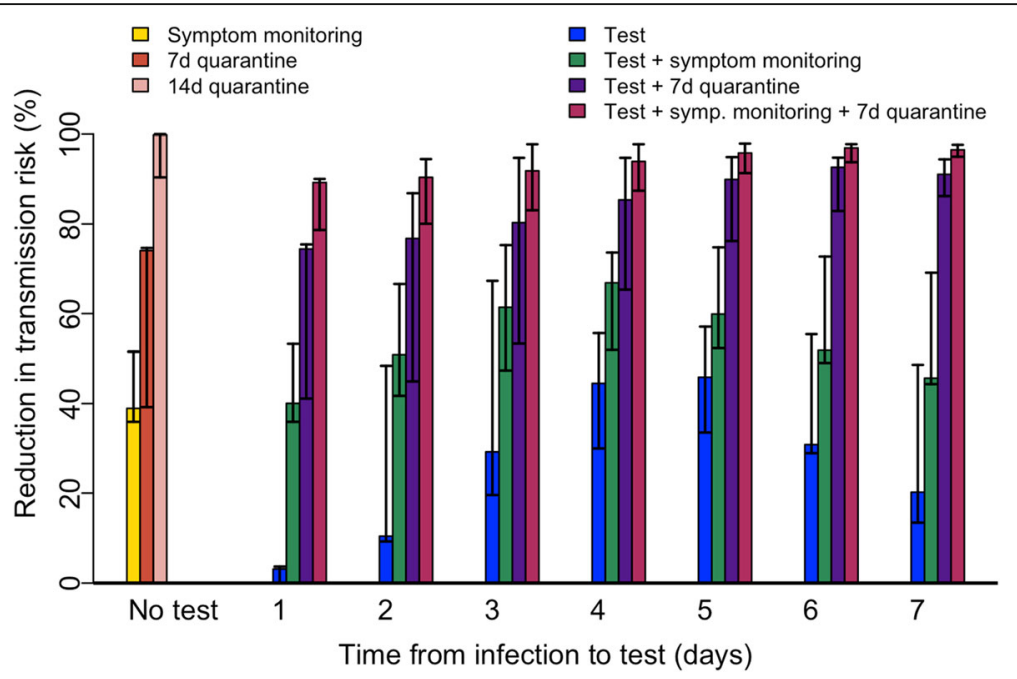

Fig. 2 Reductions in total average SARS-CoV-2 transmission risk after infection at a known high-risk exposure time (day 0) without considering travel. Transmission risk reductions are stratified by method of risk reduction including symptom monitoring, quarantine (7 or 14 days), and testing (test on days 1-7). Symptom monitoring is assumed to be ongoing regardless of the test date when implemented and either symptom onset or a positive test result is assumed to result in immediate isolation until the individual is no longer infectious. The bars represent the median estimates and the error bars show the ranges (minima and maxima) across the different infectiousness curves and test positivity curves (when testing was included)

effective at detecting infections; later testing means that while the test was more likely to be positive, the infectious period may begin prior to the test, leading to a smaller reduction in risk.

Combining symptom monitoring or quarantine with testing provided added benefit, leading to increased risk reduction, especially with a test at day $3-5$ postexposure with symptom monitoring $(47-75 \%$ reduction with $30 \%$ never symptomatic or $39-73 \%$ with $50 \%$ never symptomatic) or a test at day 5-7 with a 7-day quarantine (76-95\% reduction). A 7-day quarantine with symptom monitoring and a test at day 5-7 further increased the lower bound of likely risk reduction to $91-98 \%$ (with $30 \%$ never symptomatic, $86-97 \%$ with $50 \%$ never symptomatic). The effect of moderately different assumptions related to the proportion of infections that never result in symptoms had minimal impacts when symptom monitoring was combined with testing or quarantine, we therefore use the $30 \%$ value for this parameter in the following analyses.

\section{Transmission risk during travel}

To assess approaches for reducing risk of transmission while traveling, we assumed that exposure may have occurred at any time in the 7 days prior to departure and assessed reductions in transmission risk over a 1-day period following departure. Isolating individuals at the time of symptom onset prior to or during travel resulted in a $30-35 \%$ reduction in risk (Fig. 3a). Testing resulted in the greatest reduction of risk when the specimen was collected closest to the time of travel. Testing 3 days prior to travel resulted in a $10-29 \%$ reduction in transmission risk compared to a $44-72 \%$ reduction with testing on the day of travel. This was also true for testing combined with symptom monitoring, which had higher overall reductions.

We assessed the impact of test sensitivity relative to timing by comparing the antigen-type test model to the same model with higher sensitivity. With the same timespecific pattern but different sensitivity ( $80 \%$ vs. $95 \%$, Fig. 3b), the higher sensitivity test gives a higher reduction in transmission risk if used at the same time. However, the importance of sensitivity is intertwined with timing. The lower sensitivity test was as effective or more effective than a higher sensitivity test if it was performed closer to the time of travel. For example, the test with $80 \%$ sensitivity performed 1 day prior to departure was $47-58 \%$ effective at reducing transmission risk during travel, while the test with $95 \%$ sensitivity performed 3 days prior to departure was $18-35 \%$ effective.

\section{Transmission risk after travel}

We then considered measures to reduce the risk of SARS-CoV-2 introduction to the destination location from travelers, i.e., transmission risk after traveling (Fig. 4). Assuming infection occurs at an unknown time within a 7-day exposure period prior to arrival (i.e., including possible infection while traveling), a single test on its own was most effective when performed 1- or 2days post-arrival $(29-53 \%$ and $29-51 \%$ reduction in transmission risk, respectively). This reduction in introduction risk was higher than reductions generated by 


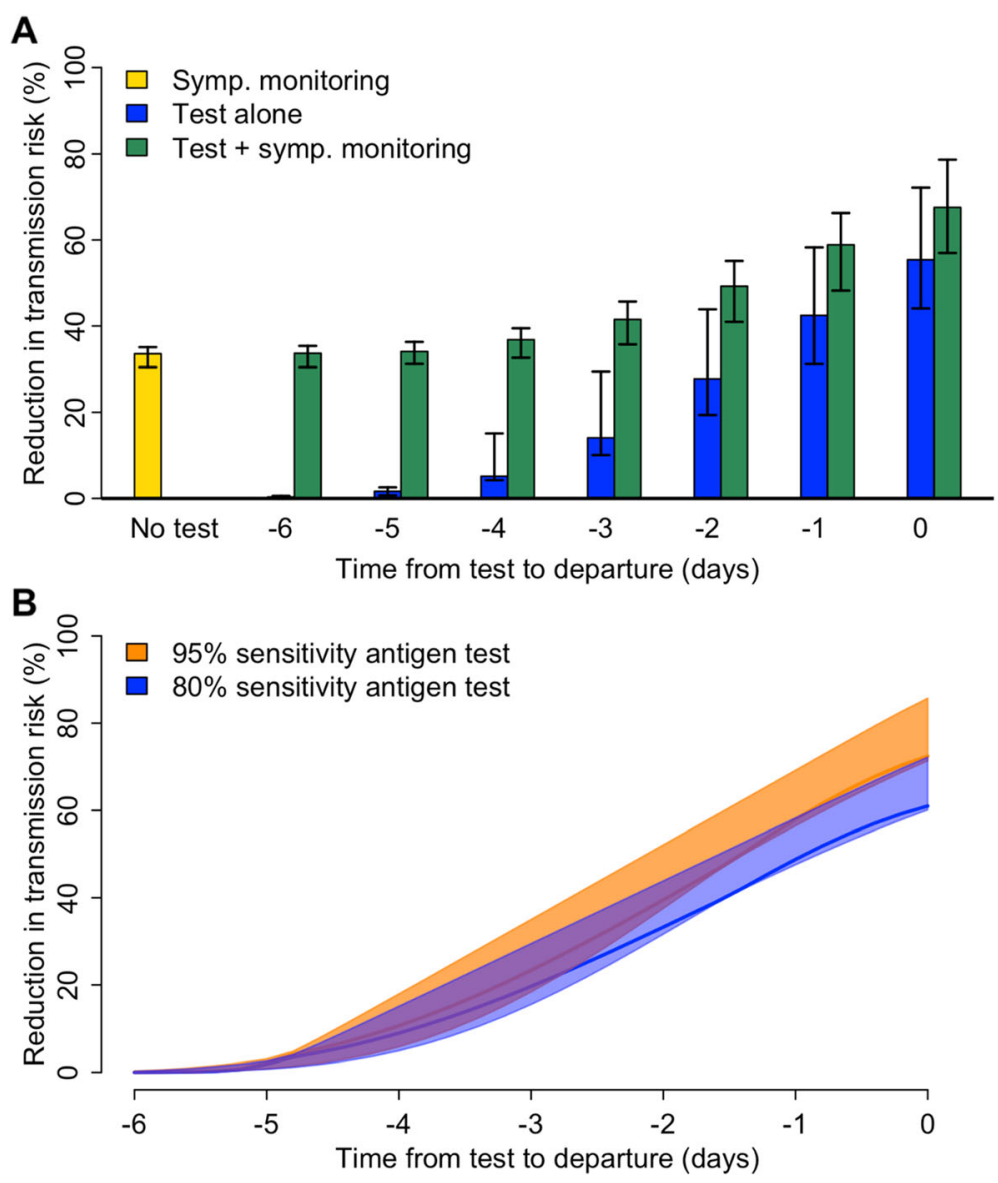

Fig. 3 Reductions in SARS-CoV-2 transmission during travel. a Reduction in transmission risk during a 1-day trip assuming a 7-day exposure window prior to travel, stratified by method of risk reduction. Individuals developing symptoms are assumed to be isolated and therefore do not travel. b Reductions in transmission risk during a 1-day trip assuming a 7-day exposure window prior to travel comparing the antigen assays with $80 \%$ and $95 \%$ sensitivity. Ranges indicate uncertainty from the different infectiousness models

testing prior to travel; a test 1 day prior to arrival provided a $17-35 \%$ reduction in risk and a test 3 days prior to arrival provided an $5-13 \%$ reduction (not shown). Tests prior to travel do not detect travelers infected while traveling and were less likely to detect travelers infected close to the time of travel. These travelers are those who are most likely to experience their entire infectious period in the destination location, and therefore, pose the greatest introduction risk.

Although a pre-travel test was less effective on its own than a post-travel test, the combination of pre-travel and post-travel tests provided additional risk reduction. A pre-travel test was most effective at reducing transmission risk after travel when performed close to the time of travel (as described above for risk during travel). In the absence of post-arrival quarantine, a second test post-travel was optimal 2-3 after arrival. The pre-travel test was likely to detect individuals who were infectious upon arrival and the later test was likely to detect those who became infectious after arrival. Combined, these tests can reduce introduction risk by $37-75 \%$. A similar effect can be attained by testing immediately upon arrival and again 2-4days post-arrival, which reduced introduction risk by $47-82 \%$.

Symptom monitoring and isolation before, during, and after travel, with no other measures in place, reduced introduction risk by $42-56 \%$ and was more effective when combined with testing (Fig. 4). For example, a test 1-day post-arrival combined with symptom monitoring before, during, and after travel reduced introduction risk by $57-75 \%$. However, quarantine for 7 days or more on its own was more effective than testing combined with symptom monitoring, regardless of when the test occurred. A 14-day quarantine reduced transmission risk by $96-100 \%$, a 10 -day quarantine by $84-100 \%$, and a $7-$ day quarantine by $64-95 \%$ (Fig. 5). Testing and symptom monitoring further enhanced the effectiveness of quarantine. A single test conducted 5-6 days after arrival with symptom monitoring and a 7-day quarantine reduced introduction risk by $97--100 \%$ (Fig. 4). The day 


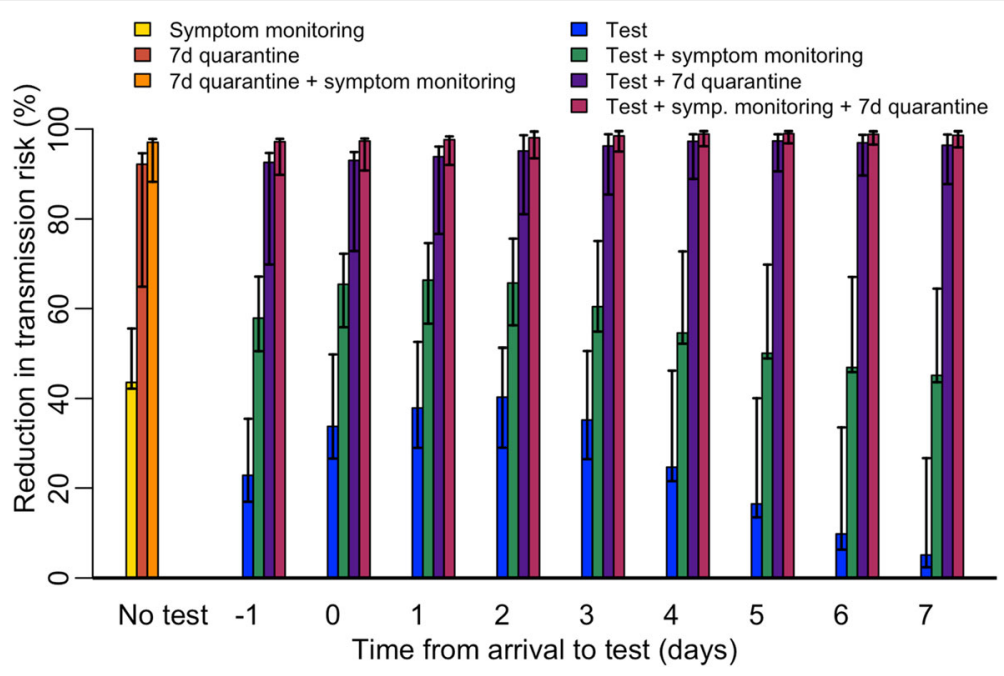

Fig. 4 Reductions in SARS-CoV-2 transmission risk from infected travelers post-arrival. Reduction in transmission risk after arrival assuming a 7-day exposure window prior to arrival, stratified by day of test and symptom monitoring, with and without a 7-day quarantine. Symptom monitoring is assumed to be ongoing before, during, and after travel and either symptom onset or a positive test result is assumed to result in immediate isolation until the individual is no longer infectious

5-6 window is optimal because it balances the reduced risk while in quarantine, with higher sensitivity for detecting individuals who may remain infectious at the end of the quarantine period.

A 7-day quarantine in conjunction with symptom monitoring and testing had similar effectiveness to a 10-day or 14-day quarantine on its own. Comparing quarantine with imperfect adherence (50\%), we found that with symptom monitoring and no test, a 7-day quarantine $(70-72 \%)$ was likely to be almost as effective as a 14-day quarantine (71-
77\%; Fig. 5). Combined with a test within 0-3 days after arrival and symptom monitoring, a 7-day quarantine with $50 \%$ adherence was estimated to be more effective (77$86 \%$ ) than a 14-day quarantine with $50 \%$ adherence and no test $(71-77 \%)$ and as effective as a 14-day quarantine with a test (77-88\%).

\section{Discussion}

Control of SARS-CoV-2 is contingent upon multiple layered mitigation measures. Reducing the risk of

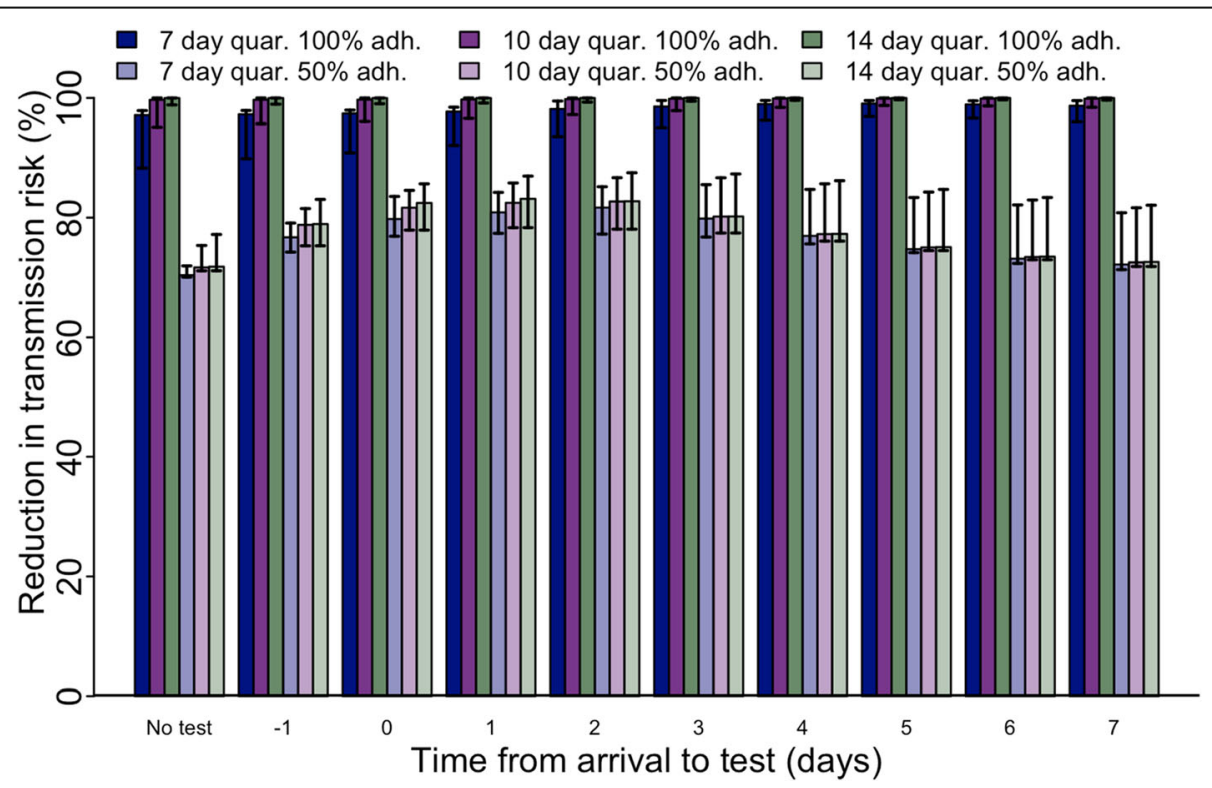

Fig. 5 Reductions in transmission risk post-arrival assuming a 7-day exposure window prior to arrival and symptom monitoring, stratified by quarantine length, quarantine adherence, and day of test 
transmission associated with travel is critical to reducing the impact related to importations on local health and healthcare systems. This is important when transmission at the destination is low and an introduction could spur additional outbreaks, but also when transmission is already high and health systems may be strained. Reducing risks associated with air travel could pave the way to air industry recovery, as well as offer relief to national economies and reduce social distress [63]. Efforts to control transmission before and after travel rely on individual mitigation measures such as mask use and social distancing before, during, and after travel, but additional control measures, such as testing and quarantine, have also been used by some countries. The fifth meeting of the International Health Regulations Emergency Committee convened by WHO regarding the COVID-19 pandemic stated that for health measures related to international travel, countries should regularly reappraise measures applied to international travel and ensure those measures (including targeted use of diagnostics and quarantine) are risk- and evidence-based [64].

Here, we used a mathematical model to assess the relative impact of three mitigation measures to reduce transmission risk from infected travelers: symptom monitoring, testing, and quarantine. We assessed combinations of these mitigation measures with different estimates of the infectious period, different estimates of test-positivity relative to time of infection, and different assumptions about infection timing and test sensitivity. We frame these results as proportional reductions in transmission risk from infected travelers during or after travel to consider the importance of optimizing mitigation measures to address peak infectiousness (Fig. 1a). On its own, quarantine was the most effective of the three strategies, with a 14-day quarantine almost eliminating risk and a 7-day quarantine being more effective than any single other measure. However, these measures can be more effective when used together. For example, symptom monitoring is relatively easy and further increases the effect of a 7-day quarantine to $88-98 \%$ with a 7-day exposure window prior to arrival (Fig. 4).

Testing also provides added benefit but is contingent on the timing and quality of the test. Testing prior to travel reduces transmission risk both while traveling and after travel if testing is done close to the time of travel. Testing closer to the time of travel is more likely to detect individuals who are infectious while traveling and immediately afterwards but can still miss infected travelers who are in their latent period, as they may not have enough viral shedding to be detected. While testing immediately prior to travel can substantially reduce risk, it poses additional logistical challenges: results must be reliably available prior to travel and protocols would be needed to effectively isolate individuals who test positive and their close contacts. On the other hand, testing more than 3 days before travel provides little benefit beyond what symptom monitoring can provide, because individuals who test positive at that point contribute less to transmission risk during and after travel than individuals who test negative because they are in their latent period or not yet infected at that time. Because of the value of testing close to the time of travel, a lower sensitivity test with faster results can be more effective despite decreased sensitivity. This finding is consistent with modeling work by Larremore et al. showing that limitations of reduced sensitivity can be overcome by more frequent testing that can still identify infections in time to reduce transmission, in this case, closer to the time of travel [65]. This conclusion draws attention to the importance of turnaround times to allow for corresponding decision-making, not just the sensitivity of the test. While test and setting-specific test turnaround times are critical to planning, they are highly varied and were not included here. These results should be considered in that context. For example, short turn-around time is very important for pre-travel testing but less critical for post-travel testing at days 3 or 4 when individuals are expected to remain in quarantine for 7 days or more.

In the absence of quarantine or with low adherence to quarantine, post-arrival testing is likely most effective 12 days after arrival, balancing early detection with optimal sensitivity for travelers in their latent period while traveling. With high-adherence quarantine or potential exposure closer to the time of travel (for example, while traveling), optimal post-arrival test timing is later, 5-6 days after travel. This corresponds to improved sensitivity for detecting individuals who may be infected close to the time of arrival and are most likely to be infectious at the end of the quarantine. With exposure up to 7 days prior to travel, we found that optimal test timing was on days $0-2$ after arrival with symptom monitoring and no quarantine, days 5-6 with symptom monitoring and quarantine with $100 \%$ adherence, and days 0-3 with symptom monitoring and quarantine with 50\% adherence. When exposure time is known more precisely or is specifically at the time of travel, for example with a high-risk contact while traveling or otherwise, the optimal test time is on days 4-5 after that exposure to optimize test sensitivity with or without symptom monitoring and on days 6-7 when combined with quarantine (Fig. 2). Beyond days 7-8 post-infection, the sensitivity for detecting infections in the models considered here begins to decrease (Fig. 1b). Even with quarantine measures in place, tests on or after arrival may have additional roles if quarantine adherence is imperfect or to assist in contact tracing when other travelers are potentially infected. Waiting to test several days after arrival improves the chance of detecting an individual who will 
be infectious at the end of the quarantine but does not optimize early detection of other infections among travelers.

These results are generally consistent with other analyses of risk associated with travel. Early in the pandemic, it was apparent that symptom screening at airports or other transit hubs could not stop the spread of SARS-CoV-2 [18]. Using an individual-level simulation framework, Clifford et al. found that more than half of infected travelers would not be detected by exit and entry screening based on temperature measurement, observation for illness, and health declaration [51]. Sufficient detection of infected travelers to avoid uncontrolled importations is largely dependent on a set of assumptions that are inconsistent with COVID-19 epidemiology: asymptomatic transmission being negligible, very high airport symptom screening sensitivity, and a short incubation period. Clifford et al. also assessed combined measures and estimated that an 8day quarantine period with an RT-PCR test on day 7 would be nearly as effective as a 14-day quarantine on its own. Other recent work highlights the effectiveness of shorter quarantine periods combined with testing for individuals with known exposures [53, 66, 67]. Across these studies, the specific days for quarantine or testing and the estimated effectiveness varied due to differences in assumptions about the time of exposure, different modeled test characteristics, and differences in parameters for the infectious period. Nonetheless, all indicate the value of shorter quarantine combined with symptom monitoring and testing, a finding that is helpful both in the travel setting and in other settings with exposure risk.

The model used here has some specific limitations. First, the infectious period of SARS-CoV-2 is not welldefined. We therefore considered multiple models of the infectious period generated by multiple approaches to reflect uncertainty around this period, yet these models also have limitations, are not exhaustive, and more detail is needed for more precise estimates. Moreover, each of the infectious period models captures only the average infectious period, so for individual travelers, this could be substantially different. The most effective measures modeled here are close to $100 \%$ effective in the model; however, the existence of individual-level variation suggests that none of these approaches would truly be $100 \%$ effective. Even with a 14-day quarantine, it is likely that some individuals will be infectious later, or even develop symptoms only at the end of the time period. Nonetheless, the average parameterization gives the expected average effectiveness for larger numbers of infected travelers; this is the scale at which policies may be most useful. Testing options are also highly varied and not well-characterized. The test options considered here are not exhaustive nor precisely characterized. Moreover, test turnaround time can also vary. We did not model test turnaround time; instead, we focused on when the test was performed, such that the result turnaround time could be considered in the context of whatever testing and laboratory resources are available. For example, a test during quarantine should be done sufficiently early so that results are available before the end of quarantine, but that delay varies in different settings. Our framework, however, can be applied with many other options, or with better characterized distributions as these become available.

We also did not consider behavioral aspects of prevention, with the exception of adherence to quarantine. For simplicity, we assumed that quarantine was equivalent to individual-level isolation and that symptomatic individuals or those testing positive are isolated immediately. However, individuals may quarantine with others. In that case, symptom onset or a positive test for a single individual can indicate exposure for the others during quarantine. Without symptom onset or a positive test, there may be silent secondary transmission that could result in additional post-quarantine risk. Moreover, travelers may have little incentive to consistently adhere to these measures, and notification or enforcement of them also would require substantial effort and resources. Some travelers could attribute symptoms to other etiologies, such as an exacerbation of a pre-existing condition or travel fatigue. Additionally, if negative test results are available prior to the recommended end of quarantine, individuals may be less likely to complete the recommended quarantine perceiving that the test is sufficient evidence of not being infected. While adherence to all measures may be lower in practice than considered here, the relative effectiveness of measures still provides a useful guide. Moreover, the effectiveness of shorter quarantines, especially when combined with symptom monitoring and testing, may be enhanced because a shorter quarantine is less onerous and may drive better adherence [68].

Finally, we focused on comparing the effectiveness of intervention measures for infected travelers, not the reduction in absolute risk as that varies by location and time. This is therefore not an analysis of the conditions in which these measures should be implemented, nor of the specific logistical and policy challenges that arise in different situations. Quarantine of all travelers can be an effective prevention measure but could also result in the restricted movement of many travelers who are not infected and, therefore, pose no risk. When the absolute risk of infection in travelers is low and the number of travelers is high, quarantine of travelers without symptoms would predominantly result in the quarantine of uninfected people. Testing is helpful in part because it 
can reduce the length of quarantine needed for optimal prevention. However, testing can also result in false negatives (missed cases that are released from quarantine when still infectious) or false positives (individuals who test positive but are not actually infected). The impact of false positives can be partly mitigated by confirmatory testing. It is also possible that some recently recovered individuals will test positive but no longer be infectious (e.g., by RT-PCR which can detect SARS-CoV-2 RNA after the infectious period has ended). Additional testing or assessment of cycle threshold values may help reduce the impact on these individuals [69]. It is important that authorities also carefully consider prioritization of testing resources in the context of other public health needs in resource-limited situations.

A multi-layered approach is needed to control SARSCoV-2 transmission associated with travel. Infection prevention measures (e.g., social distancing, mask use, hand hygiene, enhanced cleaning, and disinfection) are expected to reduce risk before, during, and after travel. Symptom monitoring, quarantine, and testing can all complement those measures to further reduce risk. Predeparture SARS-CoV-2 testing can supplement symptom monitoring to identify potentially infectious travelers who do not have symptoms, and therefore, offers an opportunity to further reduce transmission risk during and after travel. Post-arrival SARS-CoV-2 testing can identify asymptomatic or pre-symptomatic infected travelers, including some who may have tested negative prior to departure, if prior testing took place. Postarrival testing is likely effective at days $1-2$ without quarantine, but more effective later, at days 5-6, if combined with an effective quarantine of 7 days or longer. A 14-day quarantine is effective on its own but combined with testing and symptom monitoring (with isolation of those who develop symptoms or test positive), quarantine can be shortened and still be effective. These findings can inform policies for travel until safe and effective vaccines become widely available.

\section{Acknowledgements}

Not applicable

\section{Authors' contributions}

MAJ, PSD, THC, CMB, MSC, and FAR conceived the study. MAJ, HW, and PP designed and carried out the analyses. MAJ, HW, PP, PSD, and FAR drafted the manuscript. All authors revised the manuscript. The authors read and approved the final manuscript.

\section{Funding}

No specific funding source was used for this study.

\section{Availability of data and materials}

All code required to run the analysis is available at https://github.com/ cdcepi/COVID-19-traveler-model.

\section{Declarations}

Ethics approval and consent to participate

Not applicable

\section{Consent for publication}

Not applicable

\section{Competing interests}

The authors declare that they have no competing interests.

Received: 19 January 2021 Accepted: 25 March 2021

Published online: 14 April 2021

\section{References}

1. Devi S. Travel restrictions hampering COVID-19 response. Lancet. 2020; 395(10233):1331-2. https://doi.org/10.1016/S0140-6736(20)30967-3.

2. Studdert DM, Hall MA, Mello MM. Partitioning the curve - interstate travel restrictions during the Covid-19 pandemic. N Engl J Med. 2020;383(13):e83. https://doi.org/10.1056/NEJMp2024274.

3. TSA checkpoint travel numbers for 2020 and 2019 | Transportation Security Administration. https://www.tsa.gov/coronavirus/passenger-throughput. Accessed 5 Nov 2020.

4. Air travel in the time of COVID-19. Lancet Infect Dis, 2020:20:993.

5. Maneenop S, Kotcharin S. The impacts of COVID-19 on the global airline industry: an event study approach. J Air Transp Manag. 2020;89:101920. https://doi.org/10.1016/j.jairtraman.2020.101920.

6. Sun X, Wandelt S, Zhang A. How did COVID-19 impact air transportation? A first peek through the lens of complex networks. J Air Transp Manag. 2020; 89:101928.

7. Forsyth P, Guiomard C, Niemeier H-M. Covid -19, the collapse in passenger demand and airport charges 11 The authors wish to thank Brian Pearce of IATA and Michael Stanton-Geddes of ACl for helpful discussions and data, and also two anonymous referees for their comments. J Air Transp Manag. 2020;89:101932. https://doi.org/10.1016/j.jairtraman.2020.101932.

8. Airlines Seek Gate Checks for Virus to Revive Foreign Travel. Bloomberg. com. 2020. https://www.bloomberg.com/news/articles/2020-09-09/airlinesseek-u-s-airport-virus-tests-to-revive-foreign-travel. Accessed 5 Nov 2020.

9. Wilson ME, Chen LH. Re-starting travel in the era of COVID-19: preparing anew. J Travel Med. 2020;27(5). https://doi.org/10.1093/jtm/taaa108.

10. US Department of Transportation. Runway to Recovery: The United States Framework for Airlines and Airports to Mitigate the Public Health Risks of Coronavirus, Guidance Jointly Issued by the U.S. Departments of Transportation, Homeland Security, and Health and Human Services. https:// www.transportation.gov/sites/dot.gov/files/2020-07/Runway_to_Recovery_ 07022020.pdf. Accessed 5 Nov 2020.

11. Anderson SC, Mulberry N, Edwards AM, Stockdale JE, Iyaniwura SA, Falcao $\mathrm{RC}$, et al. How much leeway is there to relax COVID-19 control measures? medRxiv. 2020. doi:https://doi.org/10.1101/2020.06.12.20129833.

12. Proclamation on Suspension of Entry as Immigrants and Nonimmigrants of Persons who Pose a Risk of Transmitting 2019 Novel Coronavirus. The White House. https://www.whitehouse.gov/presidential-actions/proclamationsuspension-entry-immigrants-nonimmigrants-persons-pose-risk-tra nsmitting-2019-novel-coronavirus/. Accessed 5 Nov 2020.

13. Linka K, Peirlinck M, Costabal FS, Kuhl E. Outbreak dynamics of COVID-19 in Europe and the effect of travel restrictions. Comput Methods Biomech Biomed Engin. 2020;23(11):710-7. https://doi.org/10.1080/10255842.2020.1 759560

14. Chinazzi M, Davis JT, Ajelli M, Gioannini C, Litvinova M, Merler S, et al. The effect of travel restrictions on the spread of the 2019 novel coronavirus (COVID-19) outbreak. Science. 2020;368(6489):395-400. https://doi.org/1 0.1126/science.aba9757.

15. Pombal R, Hosegood I, Powell D. Risk of COVID-19 during air travel. JAMA. 2020;324(17):1798. https://doi.org/10.1001/jama.2020.19108.

16. International Civil Aviation Organization Council Aviation Recovery Task Force. Take-off: guidance for air travel through the COVID-19 public health crisis. 2020. https://www.icao.int/covid/cart/Documents/CART_Report_TakeOff_Document.pdf. Accessed 5 Nov 2020.

17. Mouchtouri VA, Bogogiannidou Z, Dirksen-Fischer M, Tsiodras S, Hadjichristodoulou C. Detection of imported COVID-19 cases worldwide: early assessment of airport entry screening, 24 January until 17 February 
2020. Trop Med Health. 2020;48(1):79. https://doi.org/10.1186/s41182-02000260-5.

18. Gostic K, Gomez AC, Mummah RO, Kucharski AJ, Lloyd-Smith JO. Estimated effectiveness of symptom and risk screening to prevent the spread of COVID-19. eLife. 2020;9:e55570. https://doi.org/10.7554/eLife.55570.

19. Considerations relating to passenger locator data, entry and exit screening and health declarations in the context of COVID-19 in the EU/EEA and the UK. European Centre for Disease Prevention and Control. 2020. https:// www.ecdc.europa.eu/en/publications-data/passenger-locator-data-entry-exitscreening-health-declaration. Accessed 5 Nov 2020.

20. Vilke GM, Brennan JJ, Cronin AO, Castillo EM. Clinical features of patients with COVID-19: is temperature screening useful? J Emerg Med. 2020;59(6): 952-6. https://doi.org/10.1016/j.jemermed.2020.09.048.

21. Dollard P. Risk Assessment and Management of COVID-19 Among Travelers Arriving at Designated U.S. Airports, January 17-September 13, 2020. MMWR Morb Mortal Wkly Rep. 2020;69. https://doi.org/10.15585/ mmwr.mm6945a4

22. Oran DP, Topol EJ. Prevalence of asymptomatic SARS-CoV-2 infection. Ann Intern Med. 2020;173(5):362-7. https://doi.org/10.7326/M20-3012.

23. Furukawa NW, Brooks JT, Sobel J. Evidence supporting transmission of severe acute respiratory syndrome coronavirus 2 while Presymptomatic or asymptomatic. Emerg Infect Dis. https://doi.org/10.3201/eid2607.201595.

24. Lavezzo E, Franchin E, Ciavarella C, Cuomo-Dannenburg G, Barzon L, Del Vecchio C, et al. Suppression of a SARS-CoV-2 outbreak in the Italian municipality of Vo. Nature. 2020;584(7821):425-9. https://doi.org/10.1038/s41 586-020-2488-1.

25. Buitrago-Garcia D, Egli-Gany D, Counotte MJ, Hossmann S, Imeri H, Ipekci AM, et al. Occurrence and transmission potential of asymptomatic and presymptomatic SARS-CoV-2 infections: a living systematic review and meta-analysis. Plos Med. 2020;17(9):e1003346. https://doi.org/10.1371/journa I.pmed.1003346.

26. Joshi RK, Ray RK, Adhya S, Chauhan VPS, Pani S. Spread of COVID-19 by asymptomatic cases: evidence from military quarantine facilities. BMJ Mil Health. 2020:bmjmilitary-2020-001669. https://doi.org/10.1136/bmjmilitary-2 020-001669

27. Johansson MA, Quandelacy TM, Kada S, Prasad PV, Steele M, Brooks JT, et al. SARS-CoV-2 transmission from people without COVID-19 symptoms. JAMA Netw Open. 2021;4(1):e2035057. https://doi.org/10.1001/jamanetworkopen.2 020.35057.

28. Tindale LC, Stockdale JE, Coombe M, Garlock ES, Lau WY, Saraswat M, et al. Evidence for transmission of COVID-19 prior to symptom onset. eLife. 2020; 9:e57149. https://doi.org/10.7554/eLife.57149.

29. Nishiura $H$, Linton NM, Akhmetzhanov AR. Serial interval of novel coronavirus (COVID-19) infections. Int J Infect Dis. 2020;93:284-6. https://doi. org/10.1016/j.jijid.2020.02.060

30. Zhao S, Gao D, Zhuang Z, Chong MKC, Cai Y, Ran J, et al. Estimating the serial interval of the novel coronavirus disease (COVID-19): a statistical analysis using the public data in Hong Kong from January 16 to February 15, 2020. Front Phys. 2020;8. https://doi.org/10.3389/fphy.2020.00347.

31. Wei WE. Presymptomatic Transmission of SARS-CoV-2 - Singapore, January 23-March 16, 2020. MMWR Morb Mortal Wkly Rep. 2020;69. https://doi.org/1 0.15585/mmwr.mm6914e1.

32. Tong Z-D, Tang A, Li K-F, Li P, Wang H-L, Yi J-P, et al. Potential Presymptomatic transmission of SARS-CoV-2, Zhejiang Province, China, 2020. Emerg Infect Dis. 2020;26(5):1052-4. https://doi.org/10.3201/eid2605.2 00198.

33. Considerations for quarantine of contacts of COVID-19 cases. https://www. who.int/publications-detail-redirect/considerations-for-quarantine-ofindividuals-in-the-context-of-containment-for-coronavirus-disease-(covid-19). Accessed 5 Nov 2020.

34. Peak CM, Kahn R, Grad YH, Childs LM, Li R, Lipsitch M, et al. Individual quarantine versus active monitoring of contacts for the mitigation of COVID-19: a modelling study. Lancet Infect Dis. 2020;20(9):1025-33. https:// doi.org/10.1016/S1473-3099(20)30361-3.

35. Saldaña F, Flores-Arguedas H, Camacho-Gutiérrez JA, Barradas I. Modeling the transmission dynamics and the impact of the control interventions for the COVID-19 epidemic outbreak. Math Biosci Eng. 2020;17(4):4165-83. https://doi.org/10.3934/mbe.2020231.

36. Lin C, Mullen J, Braund WE, Tu P, Auerbach J. Reopening safely - Lessons from Taiwan's COVID-19 response. J Glob Health. 10. https://doi.org/10.71 89/jogh.10.020318.
37. Lam HY, Lam TS, Wong CH, Lam WH, Leung CME, Au KWA, et al. The epidemiology of COVID-19 cases and the successful containment strategy in Hong Kong-January to may 2020. Int J Infect Dis. 2020;98: 51-8. https://doi.org/10.1016/j.ijid.2020.06.057.

38. Paltiel AD, Zheng A, Walensky RP. Assessment of SARS-CoV-2 screening strategies to permit the safe reopening of college campuses in the United States. JAMA Netw Open. 2020;3(7):e2016818. https://doi.org/10.1001/jama networkopen.2020.16818.

39. Taylor T, Das R, Mueller K, Pransky G, Christian J, Orford R, et al. Safely returning America to work: part I: general guidance for employers. Occup Environ Med. 2020;62(9):771-9. https://doi.org/10.1097/JOM. 0000000000001984.

40. Murray MT. Mitigating a COVID-19 Outbreak Among Major League Baseball Players — United States, 2020. MMWR Morb Mortal Wkly Rep. 2020;69. https://doi.org/10.15585/mmwr.mm6942a4.

41. Coronavirus (COVID-19) Travel Restrictions By Country. KAYAK. https://www. kayak.com/travel-restrictions. Accessed 5 Nov 2020.

42. Visiting Iceland. https://www.covid.is/categories/tourists-travelling-to-iceland Accessed 5 Nov 2020

43. No. 205.2: Quarantine Restrictions on Travelers Arriving in New York. Governor Andrew M. Cuomo. 2020. https://www.governor.ny.gov/news/ no-2052-quarantine-restrictions-travelers-arriving-new-york. Accessed $5 \mathrm{Nov}$ 2020 .

44. Centers for Medicare and Medicaid Services. What is CMS's policy regarding laboratories performing antigen tests authorized by the Food and Drug Administration (FDA) under an Emergency Use Authorization (EUA) for use at the point of care (POC) or in patient care settings operating under a Clinical Laboratory Improvement Amendments of 1988 (CLIA) Certificate of Waiver on asymptomatic individuals? https:// www.cms.gov/files/document/clia-poc-ag-test-enforcement-discretion.pdf. Accessed 5 Nov 2020.

45. Food and Drug Administration. Individual EUAs for Antigen Diagnostic Tests for SARS-CoV-2. FDA. 2020. https://www.fda.gov/medical-devices/corona virus-disease-2019-covid-19-emergency-use-authorizations-medical-devices/ vitro-diagnostics-euas. Accessed 5 Nov 2020.

46. Alemany A, Baró B, Ouchi D, Rodó P, Ubals M, Corbacho-Monné M, VergaraAlert J, Rodon J, Segalés J, Esteban C, Fernández G, Ruiz L, Bassat Q, Clotet B, Ara J, Vall-Mayans M, G-Beiras C, Blanco I, Mitjà O. Analytical and clinical performance of the panbio COVID-19 antigen-detecting rapid diagnostic test. J Inf Secur. 2021;0. https://doi.org/10.1016/j.jinf.2020.12.033.

47. Pilarowski G, Lebel P, Sunshine S, Liu J, Crawford E, Marquez C, et al. The CLIAHUB Consortium, DeRisi J. Performance characteristics of a rapid severe acute respiratory syndrome coronavirus 2 antigen detection assay at a public plaza testing site in San Francisco. J Infect Dis. 2021. https://doi.org/1 0.1093/infdis/jiaa802.

48. Prince-Guerra JL. Evaluation of Abbott BinaxNOW Rapid Antigen Test for SARS-CoV-2 Infection at Two Community-Based Testing Sites — Pima County, Arizona, November 3-17, 2020. MMWR Morb Mortal Wkly Rep. 2021:70. https://doi.org/10.15585/mmwr.mm7003e3.

49. Pray IW. Performance of an Antigen-Based Test for Asymptomatic and Symptomatic SARS-CoV-2 Testing at Two University Campuses Wisconsin, September-October 2020. MMWR Morb Mortal Wkly Rep. 2021; 69. https://doi.org/10.15585/mmwr.mm695152a3.

50. Burns J, Movsisyan A, Stratil JM, Coenen M, Emmert-Fees KM, Geffert K, et al. Travel-related control measures to contain the COVID-19 pandemic: a rapid review. Cochrane Database Syst Rev. 2020. https://doi.org/10.1002/14651 858.CD013717.

51. Clifford S, Quilty BJ, Russell TW, Liu Y, Chan Y-WD, Pearson CAB, et al. Strategies to reduce the risk of SARS-CoV-2 re-introduction from international travellers. medRxiv. 2020. https://doi.org/10.1101/2020.07.24.2 0161281.

52. Dickens BL, Koo JR, Lim JT, Sun H, Clapham HE, Wilder-Smith A, et al. Strategies at points of entry to reduce importation risk of COVID-19 cases and re-open travel. J Travel Med. 2020;27(8). https://doi.org/10.1093/jtm/ taaa141.

53. Ashcroft P, Lehtinen S, Angst DC, Low N, Bonhoeffer S. Quantifying the impact of quarantine duration on COVID-19 transmission. medRxiv. 2020. https://doi.org/10.1101/2020.09.24.20201061.

54. He X, Lau EHY, Wu P, Deng X, Wang J, Hao X, et al. Temporal dynamics in viral shedding and transmissibility of COVID-19. Nat Med. 2020;26(5):672-5. https://doi.org/10.1038/s41591-020-0869-5. 
55. Casey M, Griffin J, McAloon CG, Byrne AW, Madden JM, McEvoy D, et al. Presymptomatic transmission of SARS-CoV-2 infection: a secondary analysis using published data. medRxiv. 2020. https://doi.org/10.1101/2020.05.08.20094870.

56. Benefield AE, Skrip LA, Clement A, Althouse RA, Chang S, Althouse BM. SARS-CoV-2 viral load peaks prior to symptom onset: a systematic review and individual-pooled analysis of coronavirus viral load from 66 studies. medRxiv. 2020. https://doi.org/10.1101/2020.09.28.20202028.

57. Walsh KA, Jordan K, Clyne B, Rohde D, Drummond L, Byrne P, et al. SARSCoV-2 detection, viral load and infectivity over the course of an infection. $J$ Inf Secur. 2020;81:357-71.

58. Byrne AW, McEvoy D, Collins AB, Hunt K, Casey M, Barber A, et al. Inferred duration of infectious period of SARS-COV-2: rapid scoping review and analysis of available evidence for asymptomatic and symptomatic COVID-19 cases. BMJ Open. 2020;10(8):e039856. https://doi.org/10.1136/bmjopen-202 0-039856.

59. Goyal A, Reeves DB, Cardozo-Ojeda EF, Schiffer JT, Mayer BT. Wrong person, place and time: viral load and contact network structure predict SARS-CoV-2 transmission and super-spreading events. medRxiv. 2020. https://doi.org/1 0.1101/2020.08.07.20169920.

60. Wölfel R, Corman VM, Guggemos W, Seilmaier M, Zange S, Müller MA, et al. Virological assessment of hospitalized patients with COVID-2019. Nature. 2020;581 (7809):465-9. https://doi.org/10.1038/s41586-020-2196-X.

61. McAloon C, Collins A, Hunt K, Barber A, Byrne AW, Butler F, et al. Incubation period of COVID-19: a rapid systematic review and meta-analysis of observational research. BMJ Open. 2020;10(8):e039652. https://doi.org/10.113 6/bmjopen-2020-039652

62. Kucirka LM, Lauer SA, Laeyendecker O, Boon D, Lessler J. Variation in falsenegative rate of reverse transcriptase polymerase chain reaction-based SARS-CoV-2 tests by time since exposure. Ann Intern Med. 2020;173(4):2627. https://doi.org/10.7326/M20-1495.

63. Lamb TL, Winter SR, Rice S, Ruskin KJ, Vaughn A. Factors that predict passengers willingness to fly during and after the COVID-19 pandemic. J Air Transp Manag. 2020;89:101897. https://doi.org/10.1016/j.jairtraman.2020.101897.

64. Statement on the fifth meeting of the International Health Regulations (2005) Emergency Committee regarding the coronavirus disease (COVID-19) pandemic. https://www.who.int/news/item/30-10-2020-statement-on-thefifth-meeting-of-the-international-health-regulations-(2005)-emergencycommittee-regarding-the-coronavirus-disease-(covid-19)-pandemic. Accessed 5 Nov 2020.

65. Larremore DB, Wilder B, Lester E, Shehata S, Burke JM, Hay JA, et al. Test sensitivity is secondary to frequency and turnaround time for COVID-19 surveillance. medRxiv. 2020. https://doi.org/10.1101/2020.06.22.20136309.

66. Quilty BJ, Clifford S, Group2 C nCoV working, Flasche S, Eggo RM. Effectiveness of airport screening at detecting travellers infected with novel coronavirus (2019-nCoV). Euro Surveill. 2020;25:2000080.

67. Wells CR, Townsend JP, Pandey A, Krieger G, Singer B, McDonald RH, et al. Optimal COVID-19 quarantine and testing strategies. medRxiv. 2020. https:// doi.org/10.1101/2020.10.27.20211631.

68. Webster RK, Brooks SK, Smith LE, Woodland L, Wessely S, Rubin GJ. How to improve adherence with quarantine: rapid review of the evidence. Public Health. 2020;182:163-9. https://doi.org/10.1016/j.puhe.2020.03.007.

69. Bullard J, Dust K, Funk D, Strong JE, Alexander D, Garnett L, et al. Predicting infectious severe acute respiratory syndrome coronavirus 2 from diagnostic samples. Clin Infect Dis. 2020. https://doi.org/10.1093/cid/ciaa638.

\section{Publisher's Note}

Springer Nature remains neutral with regard to jurisdictional claims in published maps and institutional affiliations.

Ready to submit your research? Choose BMC and benefit from:
- fast, convenient online submission
- thorough peer review by experienced researchers in your field
- rapid publication on acceptance
- support for research data, including large and complex data types
- gold Open Access which fosters wider collaboration and increased citations
- maximum visibility for your research: over 100M website views per year
At BMC, research is always in progress.
Learn more biomedcentral.com/submissions

